\title{
Spirometric changes in bronchodilation tests as predictors of asthma diagnosis and treatment response in patients with FEV1 [?] $80 \%$ predicted
}

Huijuan $\mathrm{Hao}^{1}$, Wuping Bao ${ }^{1}$, Yishu Xue ${ }^{1}$, Yan Zhou ${ }^{1}$, Zhixuan Huang ${ }^{2}$, Dongning Yin ${ }^{1}$, Yingying Zhang ${ }^{1}$, Pengyu Zhang ${ }^{3}$, Chengjian Lv ${ }^{1}$, Lei Han ${ }^{1}$, xin Zhou ${ }^{1}$, Junfeng Yin ${ }^{2}$, and Min Zhang ${ }^{4}$

${ }^{1}$ Shanghai Jiaotong University First People's Hospital

${ }^{2}$ Tongji University

${ }^{3}$ Shanghai First People's Hospital

${ }^{4}$ Shanghai Jiao Tong University

October 1, 2020

\begin{abstract}
Background: Many patients with mild asthma are undiagnosed and untreated for low diagnostic sensitivity of the bronchodilation test (BDT). Objective: Investigating whether airway reversibility in BDT alone or together with fractional exhaled nitric oxide (FENO) can predict the response to anti-asthma therapy (RAT) in suspected asthma patients. Methods: This study included patients with chronic recurrent asthma symptoms, normal forced expiratory volume in 1 second (FEV1), and negative BDT. Inhaled corticosteroid (ICS) and long-acting $\beta$ agonist (LABA) were given for 4 weeks. Positive RAT (PRAT) was defined as improved symptoms and increase of FEV1 $>200 \mathrm{~mL}$ after ICS/LABA. Lung tissues from 19 patients with lung nodules, grouped by predicted RAT, were also analyzed. Results: Of 102 patients, the PRAT group had higher FENO and greater absolute ([?]) and ([?]\%) percent improvements of forced vital capacity, FEV1, and forced expiratory flows (FEFs) in BDT than the negative RAT group. The AUCs of FENO, [?]FEV1\%, [?]FEF25-75\%, and [?]FEF75\% for PRAT were 0.703, 0.824, 0.736 , and 0.710 , with the optimal cut-off values of $33 \mathrm{ppb}, 3.50 \%, 15.26 \%$, and $26.04 \%$. A joint model of FENO and [?]FEV1\% increased the AUC to 0.880. IL-4, IL-5, IL-13, and NF $\varkappa$ B were higher in lung tissues of patients with predicted PRAT than with predicted NRAT. Conclusion: [?]FEV1\% $>3.50 \%$ in BDT together with FENO $>33$ ppb predicted PRAT and an asthma diagnosis in patients with normal FEV1 and negative BDT. Evidence of pathological changes in the early stage of asthma increased the credibility of the predictive model.
\end{abstract}

\section{Hosted file}

manuscript 20200930.pdf available at https://authorea.com/users/362137/articles/484378spirometric-changes-in-bronchodilation-tests-as-predictors-of-asthma-diagnosis-andtreatment-response-in-patients-with-fev1-80-predicted

\section{Hosted file}

Figures.pdf available at https://authorea.com/users/362137/articles/484378-spirometricchanges-in-bronchodilation-tests-as-predictors-of-asthma-diagnosis-and-treatmentresponse-in-patients-with-fev1-80-predicted 